OP54 ABSTRACT WITHDRAWN

\section{OP55 HEALTH, HAPPINESS AND WELLBEING IN THE TRANSITION FROM ADOLESCENCE TO ADULTHOOD: APPLYING AN "EQUITY LENS" TO AN OVERVIEW OF SYSTEMATIC REVIEWS OF POPULATION LEVEL INTERVENTIONS}

${ }^{1}$ AK Macintyre*, ${ }^{2}$ J McLean, ${ }^{3} \mathrm{P}$ Campbell, ${ }^{4}$ J Williams, ${ }^{3} \mathrm{C}$ Torrens, ${ }^{5} \mathrm{M}$ Maxwell, ${ }^{2} \mathrm{H}$ Biggs, ${ }^{3}$ A Pollock, ${ }^{6}$ A Woodhouse. ${ }^{1}$ Centre for Health Policy, University of Strathclyde, Glasgow, UK; ${ }^{2}$ Research and Development, Mental Health Foundation, Edinburgh, UK; ${ }^{3}$ Nursing, Midwifery and Allied Health Professions Research Unit, Glasgow Caledonian University, Glasgow, UK; ${ }^{4}$ Clinical and Health Psychology, University of Edinburgh, Edinburgh, UK; ${ }^{5}$ Nursing, Midwifery and Allied Health Professions Research Unit, University of Stirling, Stirling, UK; ${ }^{6}$ Policy Team, Children in Scotland, Edinburgh, UK

\subsection{6/jech-2017-SSMAbstracts.54}

Background Adolescence is a key period for health and development. There is evidence for interventions to promote health in adolescence, yet health inequalities persist into adulthood. Whilst there is robust data describing socioeconomic inequalities in adult health, there is comparatively less on adolescent health, and we have little insight on what works to tackle inequities in adolescence. We aimed to apply an "equity lens" to systematic review evidence on population interventions for adolescent health. Specifically, we aimed to synthesise systematic review evidence on the equity impact of population interventions designed to improve health, happiness and wellbeing in children and young adults aged 10-24 years.

Methods A rapid systematic review of reviews (an overview) was conducted. We systematically searched the Cochrane Database of Systematic Reviews; Health technology assessments (HTA); Campbell Collaboration; EPPI; Joanna Briggs Library; and Database of Reviews of Effects (DARE), MEDLINE, EMBASE, CINAHL, PsycINFO and PubMed for systematic reviews published in English between 01 January 2005 and 07 March 2016. Two reviewers independently applied pre-specified selection criteria. Methodological quality was independently assessed using the ROBIS tool. Disagreements were resolved through discussion. Key data relating to the review focus, including information on the review aim, participants, interventions, comparisons and outcomes were extracted. Data on whether reviews reported the socioeconomic characteristics of primary studies, differential effects, or the delivery of population interventions with disadvantaged groups were also collated. Data were synthesised within evidence tables, categorised according to themes and subthemes.

Results We identified 35310 reviews, screened 1953 abstracts and retrieved 566 full text articles. 150 reviews, assessed as either low risk of bias or unclear risk of bias were included in the final synthesis. Evidence was mapped across 9 topic areas; mental health and wellbeing; tobacco free living; preventing drug abuse and excessive drinking; sexual and reproductive health; violence and abuse free living; active living; healthy eating; obesity and general health. The majority of reviews did not describe the socioeconomic characteristics of primary studies, and examination of the differential impact of population interventions varied widely across topic areas.

Conclusion Systematic review evidence on the equity impact of population interventions for adolescents is limited. To the best of our knowledge, this is the first overview to synthesise review evidence with a focus on transition from adolescence to adulthood. This work provides important insights on how we can advance a focus on equity and considers the implications for addressing inequalities in adolescent health.

\section{OP56 EFFECTS OF NATIONAL HOUSING QUALITY STANDARDS ON HOSPITAL EMERGENCY ADMISSIONS: A QUASI- EXPERIMENT USING DATA LINKAGE}

${ }^{1} S E$ Rodgers*, ${ }^{2} W$ Poortinga, ${ }^{1} R$ Bailey, ${ }^{1} R$ Johnson, ${ }^{3} F$ Dunstan, ${ }^{1} D$ Berridge, ${ }^{1} R A$ Lyons. ${ }^{1}$ Swansea University Medical School, Swansea University, Swansea, UK; ${ }^{2}$ Welsh School of Architecture, Cardiff University, Cardiff, UK; ${ }^{3}$ Institute of Primary Care and Public Health, Cardiff University, Cardiff, UK

\subsection{6/jech-2017-SSMAbstracts.55}

Background National housing quality standards are being applied throughout the UK. A housing improvement programme was delivered through a local authority to bring nearly 9000 homes up to the Welsh Housing Quality Standard (WHQS). Homes received multiple elements, including new kitchens, bathrooms, windows and doors, insulation, and heating and electrical systems, through an eight-year rolling work programme. The study aimed to determine the impacts of the different housing improvements on hospital emergency admissions for all residents.

Methods Intervention homes, council homes that received at least one element of work, were data linked to individual health records of residents. Counts of admissions relating to respiratory and cardiovascular conditions, and falls and burns, were obtained retrospectively for each individual in a dynamic housing cohort (January 2005-March 2015). The intervention cohort criterion was for someone to have lived in any one of the intervention homes for at least three months within the intervention period. Counts were captured for up to 123 consecutive months for 32009 individuals in the intervention cohort and analysed using a multilevel approach to account for repeated observations for individuals, nested within geographic areas. Negative Binomial regression models were constructed to determine the effect for each element of work on emergency admissions for those people living in homes in receipt of the intervention element, compared to those living in homes that did not meet quality standards at that time. We adjusted for background trends in the regional general population, as well as for other confounding factors.

Results People of all ages had 34\% fewer admissions for cardiovascular and respiratory conditions, and fall and burn injuries while living in homes when the electrical systems were upgraded, compared to the reference group (IRR $=0.66$, 95\% CI 0.58-0.76). Reduced admissions were also found for new windows and doors (IRR $=0.78,0.70-0.87$ ), wall insulation $(\mathrm{IRR}=0.80,0.73-0.87)$ and garden paths $(\mathrm{IRR}=0.81$, $0.73-0.90)$. There were no associations of change in emergency admissions with upgrading heating ( $I R R=0.92,0.85-$ 1.01), loft insulation, $\quad(\operatorname{IRR}=1.02, \quad 0.93-1.13)$, kitchens $(\operatorname{IRR}=1.01,0.87-1.18)$, or bathrooms $(\operatorname{IRR}=0.99,0.87-1.13)$. Conclusion Improving housing to national standards reduces the number of emergency admissions to hospital for residents. Strengths of the data linkage approach included the 
retrospective collection of complete baseline and follow up using routine data for a long-term intervention, and large scale regional adjustment.

\section{Environment 1}

\section{OP57 SOCIOECONOMIC PATTERNING OF FOOD AND DRINK ADVERTISING AT PUBLIC TRANSPORT STOPS IN THE CITY OF EDINBURGH, UK}

${ }^{1} \mathrm{~T}$ Robertson*, ${ }^{2 \mathrm{~K}}$ Lambe, ${ }^{3} \mathrm{~L}$ Thornton, ${ }^{2} \mathrm{R}$ Jepson. ${ }^{1}$ Faculty of Health Sciences and Sport, University of Stirling, Stirling, UK; ${ }^{2}$ Scottish Collaboration for Public Health Research and Policy, University of Edinburgh, Edinburgh, UK; ${ }^{3}$ Faculty of Health, Deakin University, Melbourne, Australia

\subsection{6/jech-2017-SSMAbstracts.56}

Background Outdoor advertising has been shown to disproportionately promote unhealthy foods, with links to food preferences, purchasing and consumption habits. Given the socioeconomic inequalities in food quality, consumption and obesity, it is also possible that advertisers are disproportionally advertising unhealthy food products in lower socioeconomic areas. The aim of this study was to explore the socioeconomic patterning of food advertising at bus stops in Edinburgh, UK. Our hypothesis was that there would be a higher prevalence of unhealthy (fast food, soft drinks, confectionary etc.) versus healthy (water, low sugar beverages etc.) food advertisements situated in more deprived areas within the city.

Methods This study took place in Edinburgh, Scotland in 2015. In the city, there were 2227 bus stops, 447 of which had advertising shelters (20\%). 'ODK collect', an open-source application, was used to record data at each bus stop including GPS coordinates, type of area (residential, industrial etc.), details about the food or drink product being advertised and whether a price or special offer was featured. GPS coordinates were converted to postcodes and then the area level measure of socioeconomic deprivation (Scottish Index of Multiple Deprivation; SIMD). SIMD scores were further converted into quintiles for analysis. Generalised Linear Models were used to compare the patterning of food adverts by area-level deprivation, with and without adjustment for ward size, population and area type. All analyses were conducted using SPSS version 21.

Results In total, 562 food advertisements were recorded across 298 bus stops, with a mean of 1.89 food advertisements per shelter. Over $85 \%$ of all advertisements were for food products. Nine categories of food and related advertisements were identified including alcohol, confectionary, coffee, fast food outlets, food stores, fruit juices, frozen desserts, iced coffee and soft drinks. No adverts for fresh fruit or vegetables (besides juices), water or low sugar beverages were recorded. Across all nine food categories there were no associations between increased prevalence of these adverts and deprivation level. For example, fast food outlet advertisements (which made up $39 \%$ of all food adverts) were no more likely to be present in lower versus higher socioeconomic areas $(B=0.248$, 95\% CI: $-0.082,0.578, \mathrm{p}=0.140)$.

Discussion While food advertisements were abundant across the city, there were no patterns related to the socioeconomic status of the areas where these were located. However, all could be classed as advertising unhealthy food and drink products. This study was limited by not considering other forms of outdoor advertising.

\section{OP58 EXAMINING ASSOCIATIONS BETWEEN NEIGHBOURHOOD BUILT ENVIRONMENTS AND ADIPOSITY IN THE UK BIOBANK COHORT}

${ }^{1} \mathrm{KE}$ Mason*, ${ }^{1} \mathrm{~N}$ Pearce, ${ }^{2} \mathrm{~S}$ Cummins. ${ }^{1}$ Non-Communicable Disease Epidemiology, London School of Hygiene and Tropical Medicine, London, UK; ${ }^{2}$ Social and Environmental Health Research, London School of Hygiene and Tropical Medicine, London, UK

\subsection{6/jech-2017-SSMAbstracts.57}

Background Persistent inequalities in obesity-related health outcomes may be partly due to unequal distribution of resources in local built environments. For example, differential neighbourhood access to physical activity (PA) facilities or exposure to unhealthy food environments may influence health behaviours and ultimately be reflected in uneven population distributions of overweight and obesity. Despite much research, evidence on health effects of neighbourhood environments remains inconclusive, making it difficult to generalise to suitable interventions, if indeed intervention is warranted.

Methods Using cross-sectional data from $\sim 270000$ adults aged 40-70 from the UK Biobank cohort residing across England and Wales, including linked person-centred environmental data, we examined whether features of the fast food and PA environments near an individual's place of residence were independently associated with measures of adiposity. We also constructed a composite exposure measure to examine the PA and fast food environments operating together, classifying people's neighbourhoods on a scale of obesogenicity, from high (limited PA facilities and close to a fast food outlet) to low (many PA facilities and far from a fast food outlet). Multilevel models were used to account for clustering due to an areabased sampling design and were adjusted for potential confounding effects of individual and area-level variables, including each exposure on the other.

Results Considered separately we found that greater density of PA facilities and greater distance to the nearest fast food outlet were independently associated with smaller waist circumference (WC) e.g. having $\geq 6$ formal PA facilities within a $1 \mathrm{~km}$ street network distance of home was associated with $0.91 \mathrm{~cm}$ lower WC (95\% CI: 0.32-1.49) than having no nearby PA facilities, and living $>2 \mathrm{~km}$ from the nearest fast food outlet was associated with $0.42 \mathrm{~cm}$ lower WC than living within $500 \mathrm{~m}$ (95\% CI: 0.11-0.72). Similar patterns were observed for other adiposity outcome measures. Preliminary results from ongoing analyses using the composite exposure measure indicate a possible dose response of WC to increasing combined neighbourhood obesogenicity. A series of sensitivity and other additional analyses currently underway, including the use of propensity scores, will also be presented to explore the possibility that residual confounding may explain the findings. Conclusion Combining a very large sample with wide geographical coverage and robust statistical methods we sought improved clarity on the potential health impact of two built environment exposures, and present evidence suggesting that improving neighbourhood access to PA facilities and minimising proximity to fast food outlets may help reduce adiposity in the UK mid-aged adult population. 